\title{
Clinical Outcome after Hemiarthroplasty among Patients with Fracture Neck of Femur in South Indian Population
}

\author{
Vetrivel Chezian Sengodan ${ }^{1}$, Suresh Kumar Vadivelu ${ }^{2}$
}

\begin{abstract}
Background: Femoral neck fractures in the elderly are serious injuries, often occurring in the terminal years of life and they have a major impact on society, our healthcare system, and the cost of care. It has a high incidence rate and complications. A successful surgery at the hip joint should provide a painless and stable hip. Hemiarthroplasty is the common method of treating displaced femoral neck fractures in Garden's type III and IV in the elderly. This study aims to evaluate the clinical outcome and quality of life following hemiarthroplasty.

Materials and methods: It is a retrospective study including a total of 101 patients who underwent hemiarthroplasty (cemented/uncemented) using unipolar or bipolar prosthesis due to femoral neck fracture at the Institute of Orthopaedics and Traumatology, Coimbatore Medical College Hospital, Coimbatore, during the period of 2 years between 2017 and 2019. In this analytical retrospective study, we studied several variables including age, sex, socioeconomic status, medical comorbidities, clinical outcome, postoperative complications, quality of life, frequency of follow-up, and mortality.

Results: In our study, most of the patients were in the age-group of 60-70 years with a mean age of 65 years. This study was carried out on 43 males and 58 females. There was no intraoperative complication/mortality. A total of 21 patients had complications. These were (a) posterior dislocation (3), (b) aseptic loosening (1), (c) periprosthetic fracture (1), (d) superficial infection (11), (e) deep infection (1), and other minor complications like persistent hip pain and muscle wasting. The mortality rate was about $23.7 \%$ at 1 -year follow-up.

Conclusion: The overall complications following hemiarthroplasty were acceptable. The immediate complications rate was about $8.9 \%$ and the late complication rate was about $17.8 \%$. Targeted medical intervention that focuses upon susceptible patient groups may reduce morbidity, mortality, and improve survival.

Keywords: Elderly, Femoral neck fracture, Hemiarthroplasty.

TNOA Journal of Orthopedics and Joint Surgery (2021): 10.5005/jp-journals-10079-1037
\end{abstract}

\section{INTRODUCTION}

Femoral neck fractures in the elderly are associated with high immediate and long-term postoperative mortality. ${ }^{1-3}$ Since most of the patients are fragile with severe comorbidities such as cardiovascular disease, cognitive impairment, poor pre-fracture mobility, a delayed bed to chair interval, and diabetes mellitus, management poses a challenge to the treating orthopedic surgeon. ${ }^{3,4}$ There are various modes of treatment for displaced femoral neck fractures with regard to the patient's age, functional status, and cognitive function. ${ }^{5,6}$ However, the current debate is on whether hemiarthroplasty or total hip arthroplasty is the best treatment option for the management of displaced intracapsular femoral neck fractures in elderly patients. Arthroplasty is the most common surgical treatment option currently used to restore their ability to walk and to prevent complications related to decreased ambulation following the fracture. ${ }^{5,7}$ This study aims to review the mortality and prevalence of complications following hemiarthroplasty.

\section{Materials and Methods}

A total of 101 patients underwent hemiarthroplasty due to an acute fracture of the femoral neck at the Institute of Orthopaedics and Traumatology, Coimbatore Medical College Hospital, between March 2017 and September 2019. In our institution, the treatment of acute fractures of the neck of the femur is based on the patient's age, comorbidities, walking ability, and life expectancy. Elderly patients with multiple medical comorbidities, physiological compromise, cognitive impairment, and decreased functional demands are
1,2Institute of Orthopaedics and Traumatology, Coimbatore Medical College and Hospital, Coimbatore, Tamil Nadu, India

Corresponding Author: Vetrivel Chezian Sengodan, Institute of Orthopaedics and Traumatology, Coimbatore Medical College and Hospital, Coimbatore, Tamil Nadu, India, Phone: +91 9843028096, e-mail: svcortho@gmail.com

How to cite this article: Sengodan VC, Vadivelu SK. Clinical Outcome after Hemiarthroplasty among Patients with Fracture Neck of Femur in South Indian Population. TNOA J Orth Joint Surg 2021;3(2):65-69.

Source of support: Nil

Conflict of interest: None

largely treated with hemiarthroplasty. In the healthy and active elderly with long life expectancy, total hip arthroplasty is used. Patients were initially stabilized and evaluated for an elective procedure. The positioning of the patient and approach were based on the surgeon's preference. Preoperative antibiotic prophylaxis (ceftriaxone $2 \mathrm{~g}$ IV given after test dose) was used as a single dose 30 minutes before surgery. The selection of the type of implant and bone cementing were based on the surgeon's preference and bone quality. The procedure was done by different surgeons through a posterior or lateral approach. Postoperatively, mobilization and weight-bearing were allowed as tolerated. Patients were followed periodically to evaluate postoperative complications (dislocation, periprosthetic infection, sciatic nerve palsy) and functional status of the patient. The clinical outcome of hemiarthroplasty was evaluated using HIP HARRIS SCORE. 


\section{Results}

In our retrospective analysis, 58 were females and 43 were males. The average age of females was 65.5 years. The average age of males was 64.5 years. The male:female ratio was 1:1.35. In our study the number of patients in scale 5 th was more as per modified Kuppuswamy scale (Fig. 1 and Table 1).

The mean period of delay of hospitalization for surgery was 1 week. The comorbidities were found in 53 patients, the most common being type II diabetes mellitus (52.3\%) (Table 2 and Fig. 2).

Regarding the surgical approach, the posterior approach was done for 72 patients and the lateral approach was used in 29 patients. The posterior and lateral approach ratio was 3:1. The Austin-Moore prosthesis was used in 38 cases. Bipolar nonfenestrated prosthesis with bone cementing was used in 63 cases (Fig. 3).

The duration of surgery was 60-90 minutes. No significant intraoperative complications other than blood loss were encountered. Perioperative blood transfusion was done in 67 cases. Preoperative blood transfusion was done in 32 cases to

Table 1: Modified Kuppuswamy socioeconomic scale

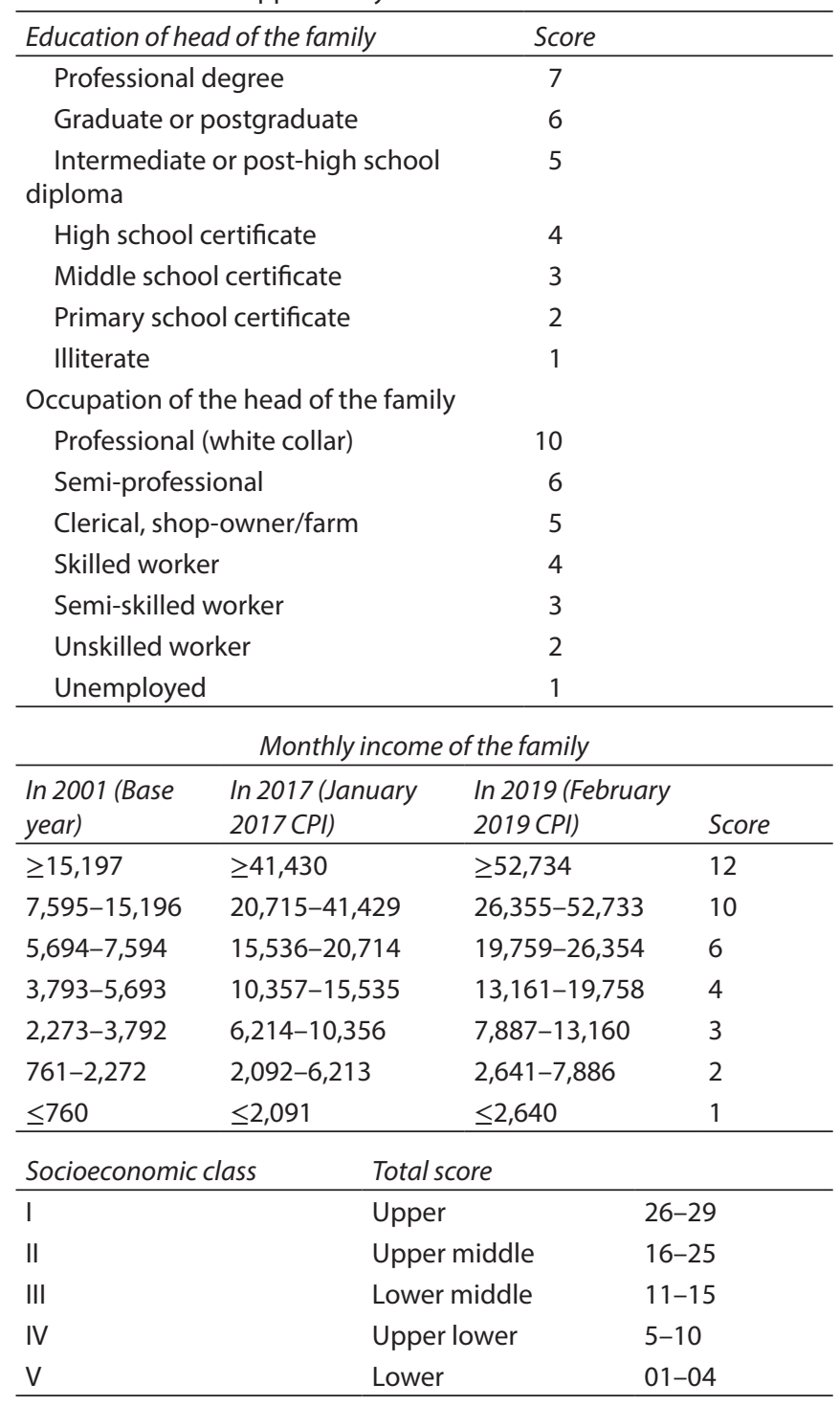

improve hemoglobin status. The mean length of postoperative stay was about 7 days. In the immediate postoperative period, we encountered complications in 10 patients which included 6 superficial infections, 2 prosthesis dislocations, 1 systemic infection, and 1 mortality and the immediate complications rate was about $9.9 \%$. The prosthetic dislocation was seen in three patients with posterior approach hemiarthroplasty who had neurological complications. In one of those patients extended trochanteric osteotomy and cerclage wiring was done as a part of the revision procedure. They were reduced under anesthesia and verified by pre- and postoperative radiographs (Fig. 4). Superficial infections were treated successfully with antibiotics, without the need for surgical debridement and re-exploration. One patient who was suffering from a chronic obstructive pulmonary disease with cardiac complication expired on the 5 th postoperative day.

The late complication rate was about $17.8 \%$. The late complications include periprosthetic fracture (1), aseptic loosening of an implant (1), prosthetic dislocation (1), persistent hip pain (5), superficial infection (6), and significant lower limb muscle wasting (4). Among these complications, persistent hip pain occurred in five patients who underwent monopolar hemiarthroplasty (AustinMoore prosthesis). Prosthetic dislocation had been reduced under anesthesia with C-arm guidance (Fig. 4).

Mortality following hemiarthroplasty was about 24 out of 101 cases. The mortality rate at a 1-year follow-up is about $23.7 \%$. During the study period, there was a loss of follow-up for 15 cases that came under Kuppuswamy classification IV and V.

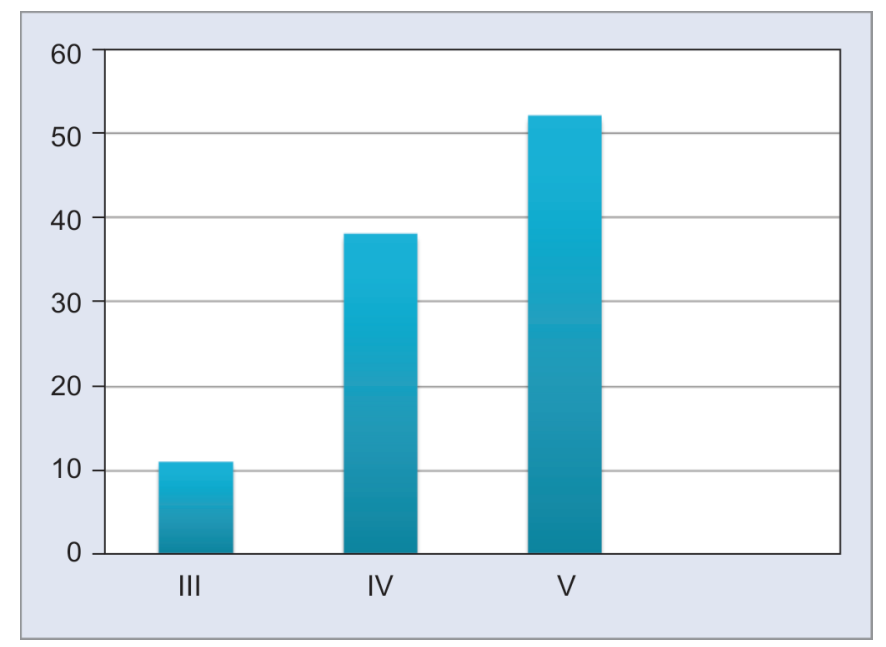

Fig. 1: Socioeconomic status of patients as per modified Kuppuswamy scale

Table 2: Demographic data demonstrating the prevalence of comorbidities among the neck of femur fracture patients in our study

\begin{tabular}{ll}
\hline Comorbidities & No. of cases \\
\hline Bronchial asthma & 02 \\
Chronic kidney disease & 04 \\
Chronic obstructive pulmonary & 03 \\
disease & \\
Diabetes mellitus & 33 \\
Hypertension & 17 \\
Epilepsy & 04 \\
\hline
\end{tabular}




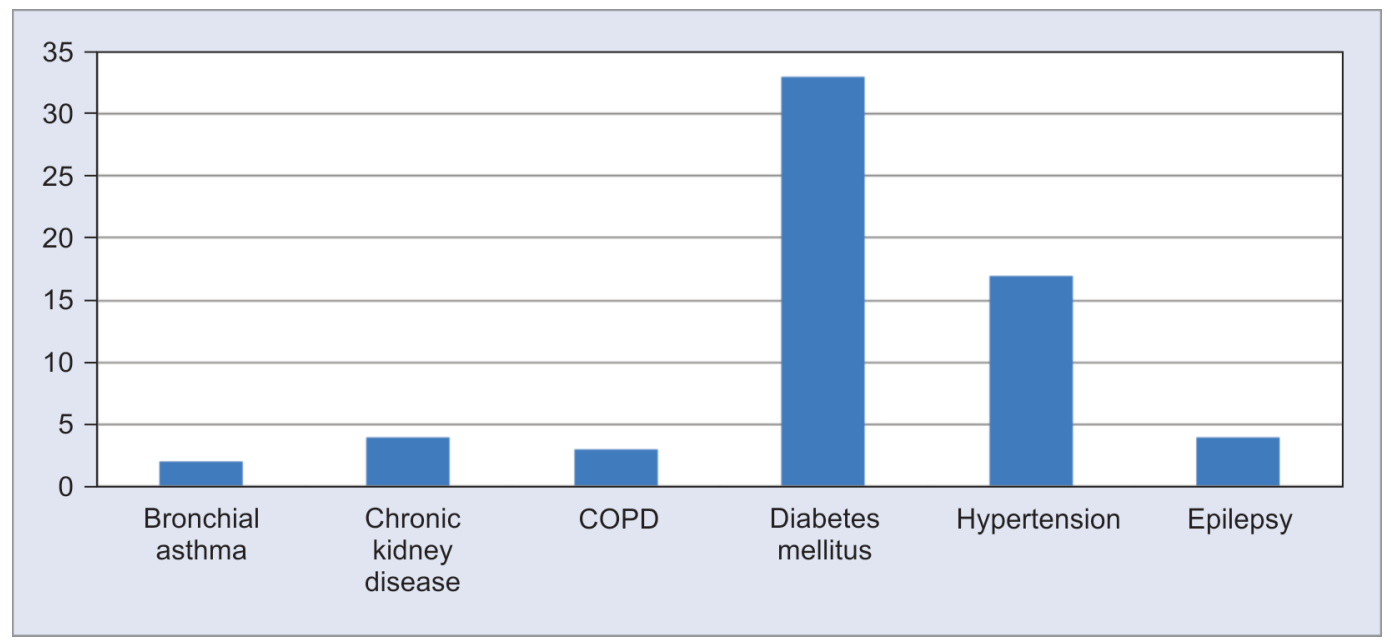

Fig. 2: Comorbidities found in our study
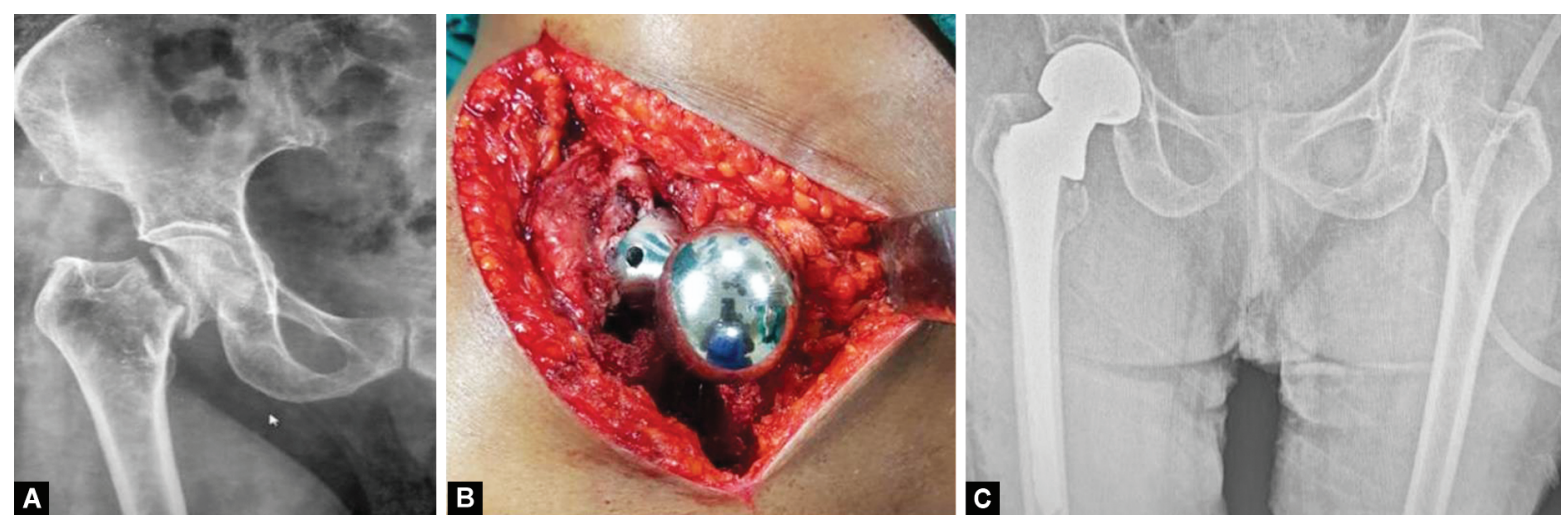

Figs 3 A to C: (A) X-ray right hip anteroposterior view showing fracture neck of femur right side; (B) Intraoperative image showing fixation of bipolar prosthesis; (C) X-ray pelvis with bilateral hips anteroposterior view showing bipolar prosthesis on the right hip
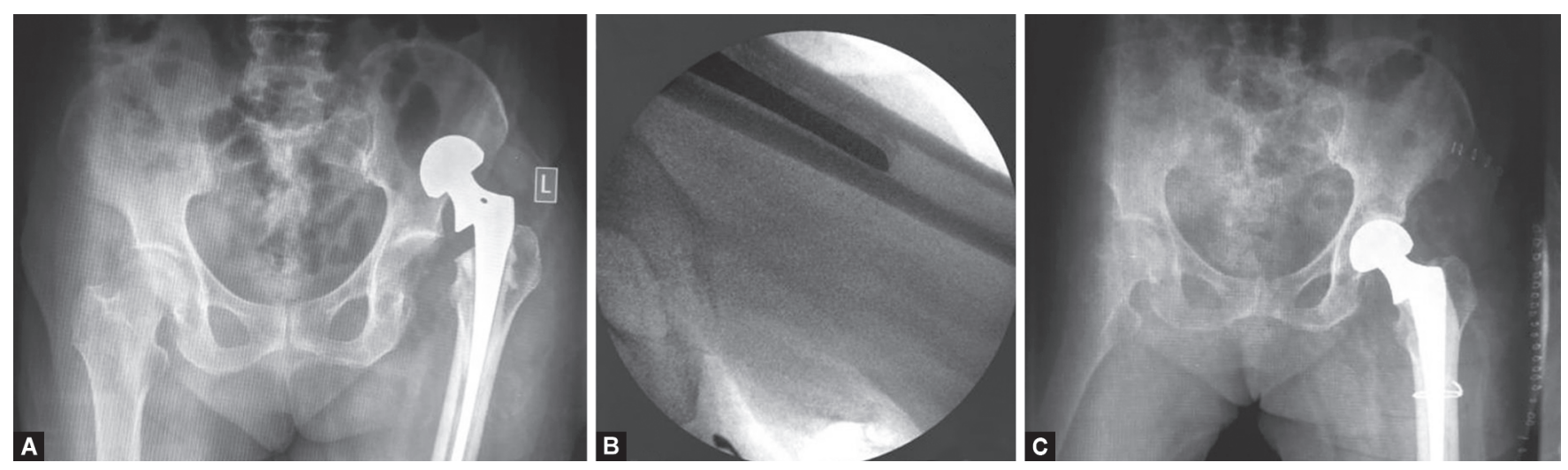

Figs 4A to C: (A) X-ray pelvis anteroposterior view showing dislocation and loosening of bipolar prosthesis left side. (B) Intraoperative fluoroscopy image showing the tip of the femoral stem; (C) X-ray pelvis anteroposterior view showing re-implantation and cerclage wiring

In our study, 24 patients died, among them, 2 and 22 patients belonged to class IV and $\mathrm{V}$, respectively. The mortality rate was higher in females.

After adding the scores given for each criterion for assessment of hip, the functional outcome was graded as excellent, good, fair, and poor. In our study, the total Harris hip score at the end of 1 year ranged from 24 to 100 (Table 3).
Thus, $51.9 \%$ had excellent results, $29.8 \%$ had good results, $9 \%$ had fair results, and $9 \%$ of the patients had poor results.

\section{Discussion}

Femoral neck fractures in the elderly have a high mortality rate. Femoral neck fractures treated with hemiarthroplasty have a decrease in mortality. ${ }^{8}$ In the present study, it was about $23.7 \%$. 


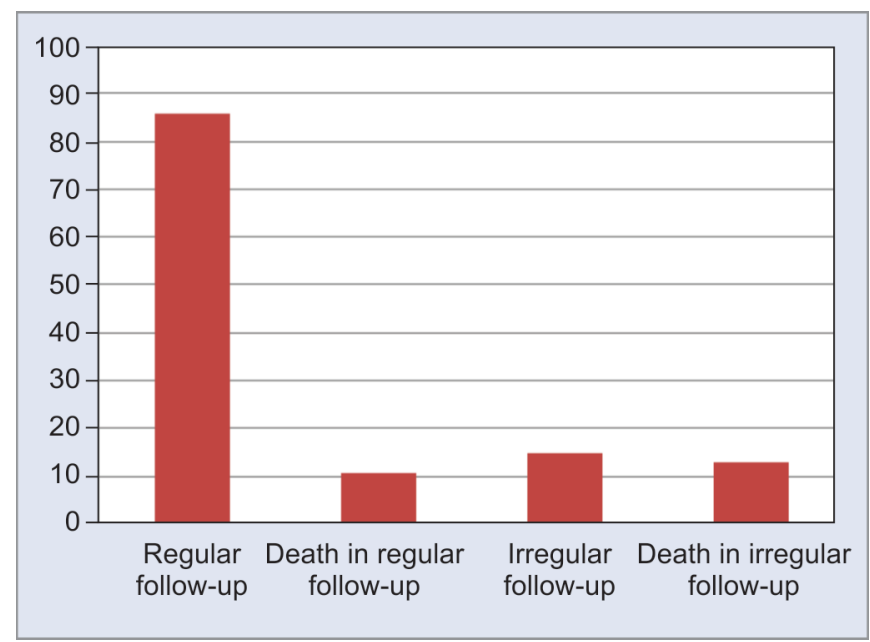

Fig. 5: Mortality status based on follow-up

Table 3: Postoperative functional outcome by Harris hip scoring system

\begin{tabular}{lc}
\hline Functional outcome & Percentage \\
\hline Excellent & 51.9 \\
Good & 29.8 \\
Fair & 9 \\
Poor & 9 \\
\hline
\end{tabular}

D'Angelo et al. reported a 25\% 1-year follow-up mortality rate following hemiarthroplasty. ${ }^{9}$ Eiskjaer reported a $20 \%$ mortality rate at 6 months and a $28 \%$ mortality rate at 1 year. ${ }^{10}$

It was found that socioeconomic status and family support play a major role in preventing morbidity and mortality. The data also suggest that patients with lower limb fractures have relatively fewer postoperative visits than patients with upper limb fractures. Most of the patients belonged to poor socioeconomic status.

Low socioeconomic status and lack of support for rehabilitation attributed to the high mortality. In our study, most of the deaths occurred in their home; hence, the cause of death could not be identified. Lack of affordability was the main factor that prevented relatives from bringing the patients for a postoperative check-up (Fig. 5). Also, the female sex had higher mortality (75\%) compared to the male sex (25\%) (Fig. 6).

Further comparative studies are needed to analyze the difference in mortality rates in THR and hemiarthroplasty. Since the advent of the procedure, hip dislocation in hemiarthroplasty has been a major concern for orthopedic surgeons. In the present study, the dislocation rate was about $3 \%$ (3 cases). In our study, an increased risk of dislocation was found in the posterior approach. Lateral approaches may reduce the incidence of posterior dislocation.

Several studies have shown the physiological and pathological effects of cementing on the cardiovascular system, such as hypoxia, severe systolic and diastolic hypotension, cardiac and lung failure. ${ }^{11,12}$ No such complications were encountered in our study. In the present study, the infection rate was about $12 \%$ and the most common risk factor being diabetes mellitus (75\%). In addition to ideal surgical practices of sterilization, routine wound care and

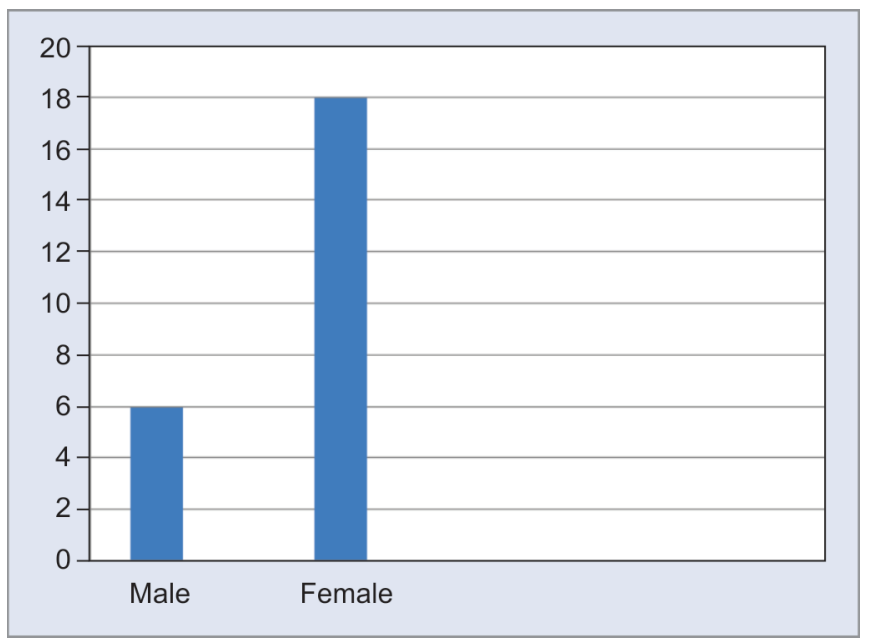

Fig. 6: Sex-wise mortality distribution

good nutritional status, and diabetic control were found to play a major role in preventing infection. ${ }^{13}$

\section{Conclusion}

In our study, the majority of the mortality had occurred within the first postoperative year. Our sample size was small, a large multicentric study is necessary to confirm our results.

\section{References}

1. Ekman E, Nurmi H, Reito A, et al. Complications following 250 cemented modular hip hemiarthroplasties. SJS Scand J Surg 2019;108(4):321-328. DOI: 10.1177/1457496918812226 journals. sagepub.com/home/sjs.

2. Smith T, Pelpola K, Ball M, et al. Pre-operative indicators for mortality following hip fracture surgery: a systematic review and meta-analysis. Age Ageing 2014;43(4):464-471. DOI: 10.1093/ageing/afu065.

3. Moja L, Piatti A, Pecoraro V, et al. Timing matters in hip fracture surgery: patients operated within 48 hours have better outcomes. A meta-analysis and meta-regression of over 190,000 patients. PLoS One 2012;7(10):e46175. DOI: 10.1371/journal.pone.0046175.

4. Keating JF, Grant A, Masson M, et al. Randomized comparison of reduction and fixation, bipolar hemiarthroplasty, and total hip arthroplasty. Treatment of displaced intracapsular hip fractures in healthy older patients. J Bone Joint Surg Am 2006;88(2):249-260. DOI: 10.2106/JBJS.E.00215.

5. Saberi S, Arabzadeh A, Khomeisi B, et al. Early complications following Bipolar hemiarthroplasty for femoral neck fracture in elderly patients. Acad J Surg 2014;1(3-4):45-48.

6. Bucholz R, Court-Brown C, Heckman J, et al. Rockwood and Green's Fractures in Adults, vol. 27 th ed., Wolters Kluwer Lippincott Williams \&Wilkins; 2010. pp. 1561-1596.

7. Schneppendahl J, Grassmann JP, Petrov V, et al. Decreasing mortality after femoral neck fracture treated with bipolar hemiarthroplasty during the last twenty years. Int Orthop 2012;36(10):2021-2026. DOI: 10.1007/s00264-012-1600-z.

8. Lim YW, Kwon SY, Han SK, et al. Postoperative mortality and factors related to mortality after bipolar hemiarthroplasty in patients with femoral neck fractures. J Arthrop 2009;24(8):1277-1280. DOI: 10.1016/j. arth.2009.06.019.

9. D'Angelo F, Giudici M, Molina M, et al. Mortality rate after hip hemiarthroplasty: analysis of risk factors in 299 consecutives cases.J Orthop Traumatol 2005;6(3):111-116. DOI: 10.1007/s10195-005-0093-6. 
10. Eiskjaer S, Østgård SE. Risk factors influencing mortality after bipolar hemiarthroplasty in the treatment of fracture of the femoral neck. Clin Orthop Relat Res 1991(270):295-300. DOI: 10.1097/00003086199109000-00039.

11. Clark D, Ahmed A, Baxendale B, et al. Cardiac output during hemiarthroplasty of the hip: a prospective, controlled trial of cemented and uncemented prostheses. Bone Joint J 2001;83(3):414418. DOI: $10.1302 / 0301-620 x .83 b 3.11477$.
12. Figved W, Opland V, Frihagen F, et al. Cemented versus uncemented hemiarthroplasty for displaced femoral neck fractures. Clin Orthop Relat Res 2009;467(9):2426-2435. DOI: 10.1007/s11999-0080672-y.

13. Antonelli B, Chen AF. Reducing the risk of infection after total joint arthroplasty: preoperative optimization. Arthroplasty 2019;1(1):4. DOI: 10.1186/s42836-019-0003-7. 